\title{
Isolated painless manual incoordination in 57 musicians
}

\author{
JONATHAN NEWMARK, FRED H HOCHBERG \\ From the Department of Neurology, Massachusetts General Hospital and Havard Medical School, \\ Boston, Massachusetts, USA
}

SUMMARY Focal motor syndromes are reported in 57 instrumental musicians who presented with painless uncoordinated movement of the upper limbs. Three stereotyped afflictions were noted: (a) flexion of the 4th and 5th fingers in pianists, (b) flexion of the 3rd finger in guitarists, and (c) extension of the 3rd finger in clarinetists. Our patients differed from those with generalised dystonia in that their disabilities were focal, activity-specific, and non-progressive. Because these disabilities represent entrained responses to peripheral stimuli, distinct from progressive dystonias, they may provide insights into control of fine limb movement and sensory triggers of abnormal movement.

Occupational cramps, also termed "craft palsies" and "occupational neuroses," have been known to physicians since Gowers published the first description in $1888 .^{1}$ Their pathogenesis and natural history remain obscure. We have seen 57 instrumental musicians with focal movement disorders amongst a population of over 450 musicians seen at the Massachusetts General Hospital for occupational upper limb complaints since 1980. A preliminary description of the patient population has been published. ${ }^{2}$ Patients with focal movement disorders presented with the chief complaint of inadequate control of the upper limb interfering with their playing. Although patients ascribed their symptoms to technical faults or fatigue, medical diagnoses had included multiple sclerosis, dystonia musculorum deformans, Parkinsonism, rheumatic diseases, or psychiatric diseases. We report the clinical appearance of isolated, single limb (often single movement) involvement by non-progressive difficulty, in the hope that we may shed light upon the causes of focal dystonic syndromes.

\section{Methods}

We retrospectively reviewed the charts of 450 musician patients seen in a 5 year period (1980-1985) at the Massachusetts General Hospital. Patients included in the study

Address for reprint requests: J Newmark, MD, Neurology, School of Medicine, University of Louisville, 319 Abraham Flexner Way, Louisville, Kentucky, 40292, USA

Received 13 December 1985 and in revised form 2 July 1986 Accepted 3 July 1986 offered a primary complaint of painless dyscontrol or inadequate coordination. We excluded patients with primary complaints of pain, trauma, paraesthesias, or truncal or global weakness. We also excluded patients whom we felt had central nervous system pathology, such as Parkinsonism, or systemic diseases such as rheumatoid arthritis.

This search yielded 59 patients. Of these, two were found subsequently to suffer from specific nerve entrapments (see below) which may have caused their symptoms, and thus were not included in the final analysis.

Twenty-two of the 59 patients were seen only once. We followed the remainder for up to 4 years, with an average follow-up time of 13 months. Follow-up was hampered by the fact that most of these patients were referred from distant centres.

\section{Illustrative case reports}

Case I A 53 year old right-handed pianist presented with a 7 year history of difficulties involving extension of the right 4th and 5th fingers. He practiced 6 to 10 hours daily, emphasising rapid scale and octave work. At age 46 he began to notice painless difficulty with right-hand octave passages. He could not clear the 4th and 5th fingers of the right hand from the keyboard. Exercises to strengthen these fingers did not affect the symptom. Colleagues began to notice his disability after 6 years. Flexion followed even light touch upon the keyboard, but was absent during all other activities, such as writing. He never complained of cramping or fatigue. At age 51 he sought examination, which was entirely normal away from the keyboard. Electrodiagnostic studies revealed normal maximum motor nerve conduction of the radial, median, and ulnar nerves, and normal F-responses and distal motor latencies of the right median and radial nerves. Conventional electromyography (EMG) of ulnar, radial, and median-innervated distal muscles was unremarkable. 
Although his flexion movement never worsened or spread beyond the 4th and 5th fingers, it remains resistant to physical therapy, biofeedback, and a trial of levodopa.

Case 2 A 51 year old right-handed professional classical guitarist experienced interphalangeal flexor tenosynovitis in his right 3 rd, 4th, and 5th fingers which completely resolved 20 years prior to his evaluation for a painless curling of the right 3rd finger into the palm. The curling, involving both metacarpal-phalangeal and interphalangeal joints, disrupted fast tremolo passages, which require rapid alternating flexion-extension movements of the 3 rd finger. The 4 th finger was slightly affected, the 5th not at all. Cortisone injection into his right 3 rd metacarpal-phalangeal joint was followed by an equally unsuccessful operative release of the right 3rd and 4th metacarpal-phalangeal flexor tendon, acupuncture, paraffin, and administration of indomethacin. Symptoms remained confined to the 3rd and 4th fingers. Upon examination, he demonstrated a resting tremor of the affected right 3rd finger and crepitus over the right 3rd proximal interphalangeal joint, indicative of prior inflammation. EMG of ulnar- and radial-innervated muscles and nerve conduction velocities of these nerves were normal. Unimproved by propranolol, he benefited from a trial of trihexyphenidyl $\mathrm{HCl}$, although his symptom persists. Despite the length of elapsed time (over 3 years), he has developed none of the stigmata of Parkinsonism such as rigidity, bradykinesia, or more gross resting tremor.

Case 3 Over a 5 year period, a 29 year old right-handed clarinetist noted the insidious onset in his right hand of painless extension of the 3rd finger coincident with 4th and occasionally 5th finger flexion into the instrument. No numbness or paraesthesia accompanied this symptom. It was involuntary and restricted to playing the clarinet; all other activities were unaffected. Braces, physical therapy, and two intrinsic-muscle release operations performed elsewhere proved useless. His examination was entirely unremarkable. We elicited his symptom immediately upon holding the clarinet, and demonstrated reproducibly that it depended upon the position of the right wrist. Maximal wrist flexion (unloading the extensor tendons) eliminated the symptom completely, as did supination of the forearm, whilst any extension of the wrist allowed the symptom to reappear. Sequential trials of trihexyphenidyl $\mathrm{HCl}$, baclofen, propranolol and amitryptıline provided no symptomatic improvement.

\section{Results}

All patients complained of technical difficulty at their instruments. Speed and volume of sound were most commonly affected. Pianists commonly experienced difficulty with ascending arpeggios; fast scale passages were most commonly affected at other instruments. Other complaints included inability to perform sustained tremolo evenly (guitar), hands rising off the instrument during slow scales (wind instruments), difficulty with crossing over of fingers (keyboard instruments), and poor general physical condition without a systemic medical illness (several). Normal activities of daily living were unaffected.

Among 59 patients primarily complaining of incoordination during playing, the age range was from 17 to 70 years, with the mean 41.7 years. Males were more often affected (42:17). Thirty-five of the 59 were keyboard players (piano, organ, harpsichord); other instruments represented in our sample included guitar and/or banjo (6), clarinet (5), violin and/or viola (4), flute (3), cello (2), bassoon (1), trumpet (1), percussion (1), and harp (1).

Difficulties evolved slowly and were of long standing; the mean duration of symptoms was 7.7 years (with a range of 4 months to 24 years). All had constant rather than intermittent symptoms. None experienced pain, paraesthesias, tremor, or numbness as a complaint. In no patient did incoordination spread beyond the hand, although in a minority activities other than instrumental playing were affected, most commonly writing and typing. None of our patients derived benefit from rest or from cessation of playing for up to years at a time.

As the three cases illustrate, patients displayed patterns of dysfunction which were often stereotyped by instrument.

(a) Flexion of the 4th and 5th fingers in pianists (Case 1). We noted painless dyscontrol of the type exemplified by Case 1 involving the 4th and 5th finger in 22 pianists. Each noted uncontrollable difficulty unrelated to trauma or medications. Twenty had involvement of the right hand, which is forced to lead in technical piano playing while simultaneously contending with the mechanical disadvantage of thinner and less sonorous strings. In every case the 4th finger was involved, although in two cases the 3rd or 5th finger was more problematic than the 4 th. Some patients eventually specialised in the meagre repertoire for left hand alone ${ }^{3}$; one became a full-time orchestral conductor. We have previously described our initial six patients with this syndrome. ${ }^{4}$

(b) Flexion of the 3rd finger in guitarists (Case 2) Four guitarists and one banjo player noted incoordination without pain upon rapid alternating flexion and extension of the right 3rd finger. One of the patients was left-handed, the others right-handed. In each, the 3rd finger was unable to extend sufficiently rapidly after plucking a string to be in position to repeat this motion. Fast scale and tremolo passages were, consequently, most severely impaired. In two patients, the problem was confined to the 3rd finger, with the 2nd and 4th finger additionally involved in each of the others. None of the patients reported spread of the problem beyond its initial location.

(c) Extension of the 3rd finger in clarinetists (Case 3) Five clarinetists experienced 3 rd finger extension and 4th and 5th finger flexion during scale passages. In four of the five, the problem was in the right hand. Scales were affected primarily, with trills (rapid alternation) a problem for all five. A postural trigger was 
seen in two of the remaining four cases in addition to Case 3. It was the impression of both patients and physicians that the primary problem was flexion of the 4th and 5th fingers, with 3rd finger extension appearing to be secondary and possibly compensatory. A postural trigger was seen in one other patient in addition to Case 3 .

Besides these three stereotyped patterns, the other 25 patients exhibited a wide variety of dysfunctions, ranging from individual finger movements to more gross hand movements such as a tendency of all fingers to flex into the palm or visible spasms of the pectoralis major muscle.

Many patients developed complicated motor strategies to cope with their difficulties. These included contraction of proximal muscles, rendering more distal fine function less dexterous; co-contraction of antagonist muscles to stabilise more distal joints, leading to fatigue and muscle pain; and more conscious use of visual cues due to subjective distrust of kinaesthesia.

In 37 of the 59 musicians we elicited histories of either trauma, inflammation, or increased demands upon the hand preceding the onset of symptoms, sometimes by years. Ten patients, adhering to the dictum "no pain, no gain," had had well-defined bouts of tendinitis or tenosynovitis in the part of the hand which later developed the present symptoms; one of these $\mathbf{1 0}$ had also had flexor retinaculum surgery. Two additional patients had undergone surgical procedures, one for "pronator syndrome" and the other unspecified, elswhere, before developing their present symptoms. Four patients had had acute injury to the hands; their injuries included one each of hyperextension of the wrist (football injury), whiplash or hyperextension of the neck (falling off a submerged $\log$ at the beach; this was the patient who later developed pectoralis spasm), finger injury in a sewing machine, and repetitive trauma inflicted by pushups and construction work. To this we added 17 patients who gave clear histories of significant increases in their practice time shortly before the onset of symptoms. The last two patients developed symptoms following the resolution of acute peripheral neuropathy in the hand, which in one case was the result of an allergic reaction to an insect bite and in the other was due to Guillain-Barré syndrome resulting from swine influenza immunisation.

Full electrophysiological testing was performed upon 36 patients, including tests of distal radial, ulnar, and median-innervated muscles and distal nerve conduction testing. These were completely negative in 31 of the 36 , some upon repeated examinations. Of the five abnormal examinations, three showed evidence of mild carpal tunnel syndrome; in none of these, however, did this evidence correlate sufficiently well with the complaints to explain the symptoms in the absence of objective sensory or motor findings upon examination. In two cases, one with EMG evidence of $\mathrm{C} 7$ radiculopathy and one with EMG evidence of mild ulnar neuropathy, the nerve compressions may have caused the observed symptoms, and these two were dropped from the original group of 59 patients.

Other laboratory tests were unrevealing. Thirteen patients had hand radiographs and seven had cervical spine radiographs, all of which were normal. Other tests included ESR and ANA (6 patients), EEG (4), CT scan of the head (3), latex fixation (3) and evoked potentials (2). All were negative except for the EEG in one patient with well documented temporal lobe epilepsy which predated the onset of her symptom.

Previous ineffective therapies included tendon release (6 patients), carpal tunnel release (3), thoracic outlet/first rib operations (2), posterior interosseous nerve release (2) and ulnar nerve release (2). Ineffective drug therapies included steroids (locally injected (7) or systemic (1)), antiParkinsonian medications (4), diazepam (3), propranolol (3), tricyclic antidepressants (2), testosterone (1) and dimethyl sulphoxide (1). Psychotherapy was prescribed by others for eight patients. Nontraditional therapies included transcutaneous nerve stimulation (3) and acupuncture (5). Fifteen patients underwent some form of physical and/or occupational therapy.

Our own attempts at treatment were only marginally more successful. Physical therapy designed to strengthen distal extensors, interossei, and lumbrical groups (usually in the 4th and 5th fingers) produced definite improvement in three patients and minimal help in five. Of a wide variety of trials of drugs, including antiParkinsonian, antidepressant, and antiGABAergic agents, only two produced significant results, trihexyphenidyl $\mathrm{HCl}$ in two patients and ethopropazine in one. Biofeedback with surface EMG electrodes produced a diminution in the sensory trigger, but no significant improvement, in Case 1.

\section{Discussion}

Since at least the time of Kinnier Wilson, ${ }^{6}$ occupational complaints of focal lack of control, such as "writer's cramp," have been considered focal dystonias of presumed central origin. ${ }^{78} \mathrm{We}$ believe that our patients may represent similar focal syndromes for which a peripheral cause appears at least as likely.

Along with dystonias, our patients' syndromes share an absence of evident denervation, sensory loss, inflammatory or compressive neuropathy, or selective weakness in a nerve or root distribution. They are equally as unresponsive to intervention by psychiatric, ${ }^{9}$ antiParkinsonian, steroidal, nonsteroidal anti- 
inflammatory, surgical, technical/occupational, and physical therapies. On the other hand, the lack of progression which our patients' aftlictions exhibited clearly separates them from the larger population of progressive dystonias, as do the lack of family history and their stereotyped relationship to activity. ${ }^{1011}$

Conditions similar to those we observed in these patients have been ascribed to psychogenic causes. Perhaps this reflects a misapprehension of the term "occupational neurosis" as originally used by Gowers; ${ }^{1}$ writing before the modern use of the term "neurosis" by Freud; Gowers did not, we believe, imply a specific psychologic mechanism by this term. We took no detailed psychiatric histories from our patients; however, in our experience, psychiatric symptoms have been rare among our musician population. Of our patients who underwent psychiatric therapy, none benefited. We have made primarily psychiatric diagnoses in none of the present series of patients and in fewer than $1 \%$ of the entire musician group of over 450 patients. $^{2}$

A subset of patients may represent subtle entrapment neuropathies which elude delineation by electrophysiologic tests. One pianist complained of dysfunction of ulnar-innervated muscles, but his neurological and electrophysiological examinations both were entirely normal. Symptom resolution followed ulnar nerve transposition. ${ }^{12}$ This case and others ${ }^{13}$ indicate that negative electrophysiological tests may not exclude peripheral pathology.

We hypothesise that occupational trauma in a subset of musicians can give rise to syndromes which may appear to be central in origin but are, in fact, purely peripheral. This concept is not original with us. Marsden et al described Sudeck's atrophy in association with abnormal muscle spasms or jerks in four post-traumatic patients. ${ }^{14}$ Even more similar to our experience is the report by Schott of segmental dystonias in four patients with recent trauma, ${ }^{15}$ in which he hypothesised that the spinal dopaminergic system may be implicated in the pathophysiology of this condition. Our patients differed from Marsden's and from Schott's only in degree; their trauma tended to be less severe and their symptoms more restricted in distribution than in Marsden's or Schott's patients. We did not examine any patients with unequivocal, more generalised dystonias. Presumably, such patients were identified and treated by others before they could be referred to us, since our patients were mostly referred after seeing multiple physicians previously.

The assumption that a central lesion, such as a small basal ganglia lacunar infarction or early Parkinsonism, had caused our patients' problems led to ineffective therapy. Published reports of such lesions have described movement dysfunctions considerably less focal than those in our patients. ${ }^{16-18}$

Our data do not suffice to allow us to form conclusions about the incidence of these problems either among musicians or among larger populations. We suspect that these problems tend not to resolve spontaneously, but our average follow-up period of 13 months did not allow confirmation of our suspicion.

Therapy for these syndromes is clearly unsatisfactory. Our patients' poor responses to centrally active drugs accords with the findings of others. ${ }^{7192021}$ Surface electrode EMG feedback in the playing position may offer a more physiologic avenue of therapy, ${ }^{22} 23$ as may attempts to diminish the overly active neuromuscular activity by selective endplate transmission blocking agents.

JN was supported by a grant from McNeil Laboratories Inc.

We thank Ching Yeh, RPT for skilled physical therapy assessment and Karen Cavanaugh for administrative assistance.

\section{References}

1 Gowers WR. Occupation neuroses. In: A Manual of Diseases of the Nervous System. Philadelphia: P Blakiston, Son \& Co, 1888:1059-78.

2 Hochberg FH, Leffert RD, Heller MD, Merriman L. Hand difficulties among musicians. JAMA 1983; 249:1869-72.

3 Strain T. Bibliography of Piano Music for the Left Hand Alone. MLS dissertation. Kent, Ohio, USA: Kent State University Press, 1972.

4 Merriman L, Newmark J, Hochberg FH, Shahani B, Leffert RD. A focal movement disorder of the hand in six pianists. Med Prob Perf Art 1986;1:17-19.

5 Fry HJH. Occupational maladies of musicians: their cause and prevention. Int J Mus Ed 1984;4:59-65.

6 Wilson SAK. Neurology (volume 2). London: Butterworth, 1940.

7 Sheehy MP, Marsden CD. Writer's cramp-a focal dystonia. Brain 1982;105:461-80.

8 Ravits J, Hallett M, Baker M, Wilkins D. Primary writing tremor and myoclonic writer's cramp. Neurology 1985;35:1387-91.

9 Roth N. Torsion dystonia, conversion hysteria, and occupational cramps. Compr Psychiatry 1980;21: 292-301.

10 Schott GD. The idiopathic dystonias: a note on their orthopaedic presentation. J Bone Jt Surg 1983;65: $51-4$.

11 Goswami U Channabasavanna SM. Transition from simple writer's cramp to dystonic writer's cramp: a report of two cases from India. Clin Neurol Neurosurg 1982;85:113-6.

12 Charness ME, Perry GJ, Marklson RE, Rosegay H, Barbaro NM. Entrapment neuropathies in musicians (abstract). Neurology 1985;35(suppl 1):74. 
13 Grevsten S, Lindsjo V, Olerud S. Recurrent ulnar nerve dislocation at the elbow: report of a non-traumatic case with ulnar entrapment neuropathy. Acta Orthop Scand 1978;49:151-3.

14 Marsden CD, Obeso JA, Traub MM, Rothwell JC, Kranz H, La Cruz F. Muscle spasms associated with Sudeck's atrophy after injury. $B r$ Med $J$ 1984; 288:173-6.

15 Schott GD. The relationship of peripheral trauma and pain to dystonia. J Neurol Neurosurg Psychiatry 1985;48:698-701.

16 Andrew J, Fowler C, Harrison MJ. Hemi-dystonia due to focal basal ganglia lesion after head injury and improved by stereotaxic thalamotomy. J Neurol Neurosurg Psychiatry 1982;45:276.

17 Traub M, Ridley A. Focal dystonia in association with cerebral infarction. J Neurol Neurosurg Psychiatry 1982;45:1073-4.

18 Narbona J, Obeso JA, Tunon T, Martinez-Lage JM,
Marsden CD. Hemidystonia secondary to localised basal ganglia tumour. J Neurol Neurosurg Psychiatry 1984;47:704-9.

19 Lang AE, Sheehy MP, Marsden CD. Anticholinergics in adult-onset focal dystonia. Can $J$ Neurol Sci 1982;9:313-9.

20 Lang AE, Sheehy MP, Marsden CD. Acute anticholinergic action in focal dystonia. Adv Neurol 1983;37:193-200.

21 Nutt JG, Hammerstad JP, Carter JH, DeGarmo PL. Lisuride treatment of focal dystonias. Neurology 1985;35:1242-3.

22 Korein J, Brudny J. Integrated EMG feedback in the management of spasmodic torticollis and focal dystonia: a prospective study of 80 patients. Res Publ Assoc Res Nerv Ment Dis 1976;55:385-426.

23 Fahn S. New approaches in the management of hyperkinetic movement disorders. Adv Exp Med Biol 1977;90:157-73. 